\title{
Transatlantica
}

Revue d'études américaines. American Studies Journal

\section{Des différents usages du terme " guerre » et de leur signification dans les représentations politiques américaines}

\section{Etienne de Durand}

\author{
(2) OpenEdition \\ Journals \\ Édition électronique \\ URL : http://journals.openedition.org/transatlantica/466 \\ DOI : $10.4000 /$ transatlantica.466 \\ ISSN : $1765-2766$ \\ Éditeur \\ AFEA
}

Référence électronique

Etienne de Durand, «Des différents usages du terme « guerre » et de leur signification dans les représentations politiques américaines », Transatlantica [En ligne], 1 | 2001, mis en ligne le 23 mars 2006, consulté le 29 avril 2021. URL : http://journals.openedition.org/transatlantica/466 ; DOI : https:// doi.org/10.4000/transatlantica.466

Ce document a été généré automatiquement le 29 avril 2021.

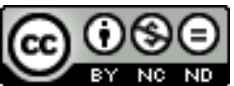

Transatlantica - Revue d'études américaines est mis à disposition selon les termes de la licence Creative Commons Attribution - Pas d'Utilisation Commerciale - Pas de Modification 4.0 International 


\title{
Des différents usages du terme " guerre » et de leur signification dans les représentations politiques américaines
}

\author{
Etienne de Durand
}

1 Lorsqu'il a déclaré engager son pays dans une "guerre contre le terrorisme », George W. Bush a tout d'abord suscité l'incompréhension et l'effroi de nombreux alliés des États-Unis, qui craignaient soit d'être entraînés dans un conflit tous azimuts contre les nombreux États ayant pratiqué ou sponsorisé le terrorisme, soit ne prenaient pas tout à fait au sérieux cette guerre d'un type nouveau. Depuis le 7 octobre, en revanche, il semble que la première guerre $\mathrm{du} \mathrm{xxI}^{\mathrm{e}}$ siècle ait pris, au moins dans sa phase initiale, des formes parfaitement reconnaissables et donc presque rassurantes. Pour autant, une certaine incompréhension transatlantique demeure, qui transparaît dans les déclarations officielles européennes : depuis le 11 septembre, les Européens éprouvent en effet des réticences à parler de "guerre", quand bien même ils s'accordent généralement à caractériser comme légitime la riposte américaine.

En marge des événements actuels, on voudrait donc s'interroger sur ce désaccord linguistique, dont la nature apparemment anecdotique traduit en réalité une différence profonde et persistante entre les cultures politiques et stratégiques des Américains et des Européens.

Bien que ce qui suit ait rarement été compris en Europe, tout du moins dans les premières semaines de la crise, le choix lexical de George $\mathrm{W}$. Bush procède de trois raisons distinctes, la première immédiatement liée aux événements du 11 septembre, la seconde enracinée dans la culture politique américaine, la dernière enfin relevant d'un choix stratégique.

La guerre de représailles, équivalence juste de l'agression

4 L'emploi du mot "guerre " par le gouvernement Bush apparaît tout à la fois comme une qualification inévitable au regard des dimensions de la catastrophe, et comme la 
réactivation volontaire de représentations historiques anciennes que le discours politique des vingt dernières années, fait de guerres métaphoriques, n'avait que partiellement recouvert.

Un désastre en mal d'équivalence autre que guerrière

5 Les attaques contre les Twin Towers et le Pentagone furent aussitôt qualifiées « d'actes de guerre » par George W. Bush. De fait, l'ampleur des dévastations perpétrées sur le sol américain justifiait certainement cette dénomination aux États-Unis mêmes, dans la mesure où elle n'ont aucun précédent dans l'histoire américaine moderne. Pour ce qui est du nombre de victimes, il faut apparemment remonter à la guerre de Sécession et à la bataille d'Antietam : ni Pearl Harbor, ni l'offensive du Têt en 1968, ni même les pires combats de la Seconde Guerre mondiale n'ont causé de telles pertes en une seule journée. En outre, et si l'on excepte les conflits avec les Indiens et en particulier les French and Indian Wars de l'époque coloniale, les États-Unis n'avaient pas connu d'attaque directe contre leur territoire continental depuis la guerre de 1812 et la capture de Washington par les Anglais en 1814 ; les rares raids aériens ou sous-marins menés par les Japonais et les Allemands furent sans conséquence. Enfin, l'Amérique n'était pas et ne se pensait pas en guerre, ce qui explique précisément les comparaisons faites avec Pearl Harbor, autre « jour d'infamie ». Etant donné la nature extraordinaire du choc et l'ampleur de l'attaque, la figure de la guerre était donc sans doute la seule qui permette de qualifier adéquatement le 11 septembre du point de vue de l'histoire et de la tradition politique américaines.

6 Parallèlement à son ampleur, la surprise de l'attaque - sa «traitrise »-et son caractère non provoqué évoquent des représentations tout à fait précises dans l'imaginaire américain. Comme l'ont remarqué plusieurs historiens et essayistes ${ }^{1}$, il existe un war narrative, c'est-à-dire une « histoire de guerre » qui se tient au cœur de la tradition historique et politique des États-Unis, et selon laquelle les représailles exercées par le peuple américain viennent toujours en réponse à une agression première et fondatrice. Ainsi au premier chef des guerres indiennes, telles qu'elles sont relatées dans la littérature américaine populaire au $\mathrm{xIX}^{\mathrm{e}}$ siècle: ce sont bien les «Peaux-rouges » qui, en enlevant et en maltraitant une femme blanche, figure même de la victime innocente, lancent un cycle qui se termine par leur écrasement aux mains de colons vengeurs ou d'une armée américaine justicière ${ }^{2}$. Si elle est loin d'être unique ou même propre aux États-Unis - les Romains eux non plus n'étaient jamais les initiateurs formels d'un conflit - cette perception de la guerre comme représailles justes faisant suite à une agression extérieure se retrouve néanmoins tout au long de l'histoire des États-Unis. A cet égard, les événements du 11 septembre ont pleinement répondu à ce "scénario " profondément ancré dans les représentations américaines, réactivé par «l'attentat " contre le Maine, à l'origine de la guerre hispano-américaine de 1898, et plus encore par Pearl Harbor : dès lors qu'il a été « lâchement frappé en premier ", le peuple américain est justifié à répliquer massivement, comme il le fit par le passé contre les "barbares", indiens, espagnols ou japonais. Dans ce cadre, la réplique ne pouvait manquer politiquement d'être à la mesure des événements, c'est-à-dire massive, visible et exemplaire, en un mot, militaire.

Significations contemporaines de la métaphore guerrière

7 Le choix d'une qualification guerrière tient également à une origine plus concrète, ancrée dans l'histoire politique des États-Unis du dernier demi-siècle. Au sein d'une structure fédérale où le terme d'Etat renvoie à des découpages administratifs et 
régionaux, et dans une société pour qui l'interventionnisme central a souvent été répudié comme un échec économique ou un empiètement politique, le gouvernement «national », c'est-à-dire fédéral, n'est jamais aussi légitime que lorsqu'il agit justement dans le domaine de la sécurité nationale - à vrai dire son occupation principale depuis la fin de la Seconde Guerre mondiale et pendant toute la guerre froide, dès lors que l'on met de côté les divers programmes de la Great Society. Remarquons d'ailleurs que ce n'est que dans le domaine de la sécurité que l'action du gouvernement est dite nationale, les autres domaines étant du ressort des institutions et administrations fédérales. Dans le même esprit, seules les forces armées américaines ont le privilège d'être qualifiées de nationales.

Outre ces divers héritages, les États-Unis ont été également marqués par une histoire récente encombrée de guerres métaphoriques et symboliques ${ }^{3}$. Depuis la fin de la guerre du Vietnam, au fur et à mesure que la population montrait un bien faible appétit guerrier et que les interventions militaires des États-Unis étaient pour la plupart extrêmement limitées, bref au fur et à mesure que la guerre réelle s'éloignait, la métaphore de la guerre se trouvait de plus en plus fréquemment utilisée pour désigner des combats politiques censés impliquer une forte mobilisation populaire à l'échelle de la nation entière. Les années 80 et 90 ont ainsi vu se succéder la "guerre contre le crime » et la "guerre contre la drogue » en plus de la gender war - quelle qu'ait été par ailleurs l'étendue réelle des mesures ou des polémiques ainsi désignées. Au final, la "guerre ", dans la culture politique contemporaine aux États-Unis, désigne le domaine d'action par excellence du gouvernement en même temps qu'elle évoque et favorise la mobilisation nationale.

9 Depuis l'attaque félonne jusqu'aux représailles exercées à l'encontre du régime des Taliban, et malgré le caractère proprement inouï des attentats de New York et Washington, le 11 septembre en est ainsi venu à dessiner une figure familière sur l'arrière-fond des représentations traditionnelles et du discours politique récent aux États-Unis.

La guerre, paradigme de la mobilisation intérieure

Dans la continuité de cet arrière-fond symbolique et politique, le paradigme de «la guerre " a pour fonction intérieure de pérenniser l'unité nationale produite par les attentats; mettre l'Amérique sur le pied de guerre permet en effet de mobiliser l'opinion publique et donc de s'assurer que le ralliement bipartisan du Congrès est durable.

11 L'équivalence posée par George W. Bush entre le 11 septembre et des actes de guerre n'est bien entendu pas restée sans conséquence en termes de politique intérieure. D'après les premiers sondages effectués immédiatement après les événements, la population américaine, à hauteur de $70 \%$ ou $80 \%$, réclamait ainsi des représailles de grande ampleur. Une fois passées les célébrations de deuil et l'attente concernant d'éventuels survivants à la tragédie de New York, le gouvernement Bush était donc appelé à subir une pression politique grandissante en faveur d'une réponse militaire. Etant donnée l'énormité du bilan humain et matériel, il eût d'ailleurs été extrêmement difficile pour n'importe quelle Administration, républicaine ou démocrate, de prétendre s'en tenir à une approche " policière » visant à arrêter les responsables. Dans ce contexte, le recours à une solution militaire est donc apparu comme une impulsion irrésistible, que le gouvernement Bush s'est toutefois efforcée de canaliser dans le sens d'une lutte de longue haleine. 
12 Parce que le 11 septembre a en quelque sorte réactivé des représentations traditionnelles, il s'est en effet agi pour le gouvernement, du moins à court terme, de canaliser la mobilisation et les attentes de la population afin d'obtenir un consensus politique large et durable, tout en évitant de céder à la tentation de représailles tous azimuts, aussi dévastatrices que potentiellement contre-productives. Compte tenu du contexte international, en particulier des sensibilités du monde arabo-musulman, il était ainsi impensable que les États-Unis mettent réellement en pratique, sur le modèle des «tuniques bleues» face aux « Peaux-rouges», une attaque simultanée contre tous les sanctuaires terroristes.

13 En ce sens, l'emploi du terme guerre et les images tirées du folklore américain - le fameux dead or alive - ne devaient s'entendre ni comme des rodomontades de circonstance, ni strictement au pied de la lettre : elles se voulaient tant un signe de détermination sur le front intérieur qu'une indication claire pour le reste du monde que les États-Unis n'hésiteraient pas à s'engager, le cas échéant, dans des actions militaires. Dans la foulée des attentats, le langage de guerre a ainsi permis la réalisation d'une « union sacrée " politique, le Congrès donnant pratiquement carte blanche à George Bush pour son action internationale dans les semaines à venir. Incidemment, se placer d'emblée sur le terrain de la guerre a permis au gouvernement Bush de couper court aux critiques et aux menaces habituelles en provenance des deux Chambres : une fois la guerre politiquement déclarée, et eu égard aux circonstances, il était extrêmement difficile pour le Congrès d'essayer d'influencer l'Administration en brandissant le «War Powers Resolution» comme à l'accoutumée en cas "d'intervention ». Dans un premier temps, le Congrès en a donc été réduit à prendre des mesures ponctuelles d'aide et à s'atteler aux réformes structurelles nécessaires (justice, renseignement), non sans toutefois que commencent à émerger des signes de divisions politiques, particulièrement à propos de la question des libertés et des droits individuels.

La guerre, option privilégiée de la stratégie américaine de " désanctuarisation »

14 Comme les responsables américains le laissaient cependant entendre dès les tous premiers jours de la crise, et comme le début de l'offensive en Afghanistan l'a confirmé, l'emploi délibéré et répété de l'image de la guerre avait également pour fonction de préparer l'opinion publique à des opérations militaires bien réelles. Pour cette raison, il serait certainement erroné de laisser entendre que le recours à l'option militaire contre Al Qaïda a majoritairement tenu à une sorte de conditionnement politique et culturel, qui aurait fait que les États-Unis, au niveau national et gouvernemental, étaient incapables de répondre autrement que militairement. Bien qu'elle s'appuie sur des représentations et un discours politique préexistant, l'action engagée en Afghanistan procède en effet d'un choix stratégique, lui-même déterminé à la fois par la posture adoptée par Al Qaïda et par la valeur dissuasive qu'est censé avoir pour l'avenir le renversement du régime des Taliban.

15 Si l'impératif politique immédiat d'une réponse apparente et donc militaire ne faisait ainsi guère de doute, la politique intérieure n'était pas seule en cause : les opérations engagées en Afghanistan visaient d'abord à répondre à la stratégie d'Al-Qaïda. Selon toute vraisemblance, Oussama ben Laden et ses lieutenants avaient en effet délibérément choisi d'attirer les États-Unis en Afghanistan, afin de cristalliser aux yeux $\mathrm{du}$ monde entier une lutte en cours depuis presque dix ans, afin également de bénéficier de conditions aussi favorables que possible. Politiquement et militairement, 
l'Afghanistan constituait en effet un théâtre d'opérations presque idéal, tant par sa géographie, véritable cauchemar de logisticien, que par un cadre politique interne et régional fait d'ambitions de puissance, d'antagonismes personnels et d'oppositions de toute nature. En obligeant les États-Unis à intervenir dans un tel contexte, les dirigeants d'Al-Qaïda espéraient sans doute tout à la fois enflammer les populations musulmanes de par le monde, et singulièrement les Pachtounes du Pakistan, et entraîner l'armée américaine dans un bourbier où les rigueurs du terrain et du climat, l'hostilité de la population et le soutien discret de certaines puissances étrangères auraient entraîné des pertes humaines nombreuses et, à terme, le découragement de la population américaine ou la révolte des populations musulmanes.

Que les événements aient apparemment pris un tour différent s'explique sans doute par le fait que ben Laden et ses hommes ne pouvaient guère que choisir le terrain de l'affrontement, et non en déterminer les termes: les États-Unis sont parvenus, difficilement il est vrai, à doser adéquatement la "carotte » et le "bâton", tantôt cajolant et aidant les pays frontaliers - dont le soutien était indispensable - et les opposants aux Taliban, tantôt les contraignant par la menace. A cette occasion, et suite à des débats internes houleux entre le Département d'Etat et le Pentagone, les États-Unis ont réussi, en quelque sorte contre leur propres traditions militaires ${ }^{4}$, à livrer avec succès ce qui ne pouvait qu'être qu'une guerre limitée, compte tenu des circonstances locales et régionales.

Outre le contexte politique qui l'obligeait à répliquer militairement sur le terrain choisi par son ennemi, le gouvernement Bush a pris, apparemment très tôt, la décision consciente de relever le défi en Afghanistan, dans le double but de mettre un terme au sanctuaire que représentait ce pays pour l'organisation de ben Laden et, plus généralement, de faire la démonstration qu'aucun sanctuaire ne tiendrait face à la détermination américaine. Par-delà Al-Qaïda, en effet, la «désanctuarisation» du «terrorisme à portée globale » semble bien avoir été l'idée directrice de l'équipe Bush, ce qui permet de mieux comprendre des expressions comme war on terrorism ou ending states that sponsor terrorism, c'est-à-dire mettre fin aux régimes, et non à proprement parler aux États, qui abritent et utilisent les réseaux terroristes. L'histoire dira si le projet américain est appelé à se poursuivre au-delà des seuls Taliban. Si les déclarations initiales des membres les plus véhéments de l'Administration, comme Paul Wolfowitz, n'ont pour l'heure pas été suivies d'effet, rien n'assure que les États-Unis ne décident pas d'avoir recours, dans un futur plus ou moins proche, à l'option militaire, en particulier si certains États ou régimes se révèlent soit parfaitement sourds aux demandes de Washington, soit trop impliqués dans le terrorisme.

18 A l'encontre de l'usage européen, qui tend à réserver le terme de "guerre » aux seuls conflits de grande ampleur opposant des rivaux de rang égal, le gouvernement Bush a proclamé, contre le terrorisme, une guerre d'un genre inédit à partir de représentations anciennes et de détournements métaphoriques plus récents. De la sorte, elle est parvenue jusqu'ici à mobiliser son opinion dans le sens d'un engagement maîtrisé et durable, qui a reçu une première application avec les opérations bien réelles conduites en Afghanistan. En marge des répercussions objectivement considérables qu'il a déjà eu et va continuer d'avoir sur la scène intérieure américaine comme sur les relations internationales, le 11 septembre a donc illustré une fois encore l'importance du choix des termes et la persistance de malentendus culturels entre les deux rives de l'Atlantique. 


\section{BIBLIOGRAPHIE}

Quelques références pour poursuivre la réflexion sur la stratégie et la politique de défense des Etats-Unis :

Jean Heffer, « Les grandes représentations stratégiques », Le débat stratégique américain 1994-95, Cahier d'Etudes Stratégiques $\mathrm{n}^{\circ} 18$ (EHESS-GSD, 1995).

Think Tanks

Center for Defense Information : http://www.cdi.org

Commonwealth Institute : http://www.comw.org/pda/milbkmrk.html

Center for Strategic and International Studies : http://www.csis.org

Council on Foreign Relations : http://www.cfr.org

National Institute for Public Policy (Républicain) : http://www.nipp.org

The Brookings Institution : http://www.brook.edu

Revues

Foreign Affairs : http://www.foreignaffairs.org

Foreign Policy : http://www.foreignpolicy.com

The Washington Quarterly (CSIS) : http://www.twq.com

\section{NOTES}

1. Cf. en particulier Michael Sherry, In the Shadow of War: the United States since the 1930's (New Haven : Yale University Press, 1995), ainsi que Tom Engelhardt, The End of Victory Culture: Cold War America and the Disillusioning of a Generation (New York : Basic Books, 1995).

2. Voir Engelhardt, The End of Victory Culture, chap. 2, "Story Time", 16-36.

3. Voir encore sur ce point Sherry, In the Shadow of War.

4. Cf. Russell F. Weigley, The American Way of War - A History of United States Military Strategy and Policy, Wars of the United States Series (Bloomington : Indiana University Press, 1977) (édition originale : Macmillan, 1973). Ce classique de la littérature stratégique américaine explique ainsi, à raison, que les États-Unis ne sont jamais aussi à l'aise que lorsqu'ils peuvent conduire des grandes guerres appuyées sur la mobilisation humaine et industrielle du pays, bref des guerres totales, sur le modèle de la Seconde Guerre mondiale. 


\section{AUTEUR}

\section{ETIENNE DE DURAND}

Etienne de Durand est chercheur au CLESID (Université Lyon 3) et à l'IFRI. Il a été Visiting Fellow à l'Université Harvard et au MIT. Il prépare une thèse sur « la culture américaine de la victoire » sous la direction d'Alain Joxe à l'EHESS. 